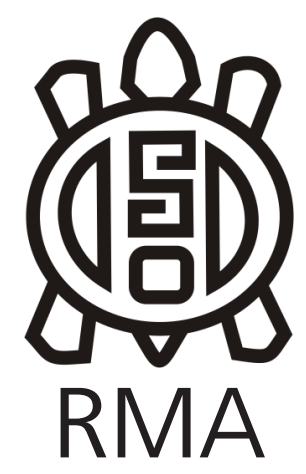

Dossier

\title{
Reflexiones para la interpretación de marcadores óseos de actividad basadas en un estudio cinemático experimental de actividades mineras prehispánicas
}

\author{
Reflexions for the interpretation of bone activity markers based on an \\ experimental cinematic study of prehispanic mining activities
}

Josefina Urrea-Navarrete; Pedro Andrade; Sonia Parra; Oscar Achiardi; Hernán Salinas y Mary Anne Argo

Universidad de Concepción, e-mail: jourrea@udec.cl; pandradem@udec.cl; soniparrao@ gmail.com; oscar.achiardi@pucv.cl; salinas.williams@gmail.com; margo@udec.cl

\begin{abstract}
Resumen
Los marcadores óseos de actividad física han sido ampliamente utilizados para la reconstrucción de actividades en las poblaciones pasadas. Este estudio compara la variabilidad de la activación muscular que existe en la ejecución de posibles gestos técnicos utilizados en las tareas de extracción y procesamiento de óxidos de hierro en Taltal, en base a los antecedentes del registro arqueológico material y experimental. Se midió la actividad muscular por medio de electromiografía superficial de diez movimientos en diferentes posturas, generados por dos gestos técnicos de extracción de pigmentos minerales y un gesto técnico de molienda. La muestra se conformó por 16 sujetos. Se registró la actividad de los músculos deltoides (porción anterior, media y posterior), trapecio superior, serrato anterior, pectoral mayor (porción horizontal y oblicua), bíceps braquial, tríceps braquial, flexor radial del carpo, extensor radial del carpo. Los resultados corresponden a las descripciones y comparación de la activación muscular de los movimientos replicados. Nuestro trabajo evidenció algunas limitantes que se podrían manifestar durante la interpretación de los marcadores óseos de actividad para poblaciones pasadas, así como la necesidad de realizar estudios experimentales y desarrollar metodologías más específicas que permitan precisar la interpretación.
\end{abstract}

Palabras claves: Bioarqueología; Reconstrucción de Actividades; Minería; Norte de Chile; EMG

\begin{abstract}
The physical activity bone markers have been a widely used to the reconstruction of ancient populations activities. This study compares the muscular activation variability that exists in the probable gestures produced during the iron oxide extractive and processing activities in Taltal, taking in consideration the archaeological material registry and experimental background. This study measured the muscular activity through superficial electromyography analysis of ten different movements performed in various postures: two technical mineral pigment extraction gestures and one grinding technical gesture. The sample for this investigation were 16 subjects, whose muscular activity was measured while performing this gestures. The measured muscles where the deltoid (anterior, medial and posterior portions), trapezius (superior portion), serratus anterior, pectoralis major (oblique and horizontal portions), biceps brachii triceps brachii, , flexor carpi radialis and extensor carpi radial. The results correspond to ten kinematic descriptions of the muscular activation involved during the replicated movements. Our work evidence some interpretation limitations when investigating activity bone markers as well as the necessity of performing experimental studies and development methodologies, which allow to clarify the interpretation.
\end{abstract}

Keywords: Bioarchaeology; Reconstruction of activities; Mining; Northern Chile; EMG

Los marcadores óseos de actividad (MOA) corresponden a cambios morfológicos que se observan en la estructura interna y externa del hueso seco, incluyendo tanto al tejido óseo en sí mismo, como también las huellas de estructuras que estuvieron asociadas a éste (Dutour 1992). Los MOA han sido interpretados como reflejo de una respuesta de adaptación fisiológica del tejido óseo a las cargas mecánicas (Kennedy 1989). A partir de antecedentes generados por la medicina deportiva, los estudios del trabajo y enfermedades laborales actuales o de colecciones osteológicas documentadas, se han vinculado ciertos cambios a nivel osteológico con determinados patrones de actividades y/u ocupaciones (Capasso et al. 1999; Dutour 1992; Kennedy 1989).

Las principales categorías de indicadores/marcadores de patrones de actividad a nivel óseo son cambios a nivel osteológico agrupados en Osteoartritis, Inserciones Musculares (Cambios Entésicos) y Geometría de la Sección Transversa del Hueso (Jurmain et al. 2012). 
De igual manera, algunas morfologías distintivas y/o alteraciones patológicas resultado de actividades laborales han sido descritas como Marcadores de Estrés Ocupacional (MOS, Markers of Ocupational Stress) (Capasso et al. 1999). Sin embargo, se reconocen que todos éstos no están directamente relacionados con una influencia primaria de actividades (Jurmain et al. 2012) dado que la respuesta del tejido óseo frente a los estímulos es compleja y depende de la interacción de varios factores fisiológicos y biomecánicos, así como también patológicos que pueden afectar su morfología (Jurmain et al. 2012; Villotte y Knüsel; 2012, Weiss 2003; Weiss et al. 2012, Wilczak 1998).

Los principales problemas metodológicos, en contextos arqueológicos, para establecer un vínculo entre el marcador y la actividad, de acuerdo a Dutour (1992) son: la especificidad del marcador, validación del indicador con estudios actuales, la transposición del gesto técnico y marcador (con real correspondencia entre la actividad actual con la pasada), cinesiología de la actividad pasada para su recreación experimental. Por otra parte, Jurmain y colaboradores (2012) sintetizan y evidencian los múltiples problemas metodológicos que conlleva la interpretación de MOA, recalcando que el vínculo entre el/los indicador/es y una actividad es difícil y complejo de establecer, no sólo desde el punto de la respuesta biológica del hueso, sino de la asociación del mismo con una actividad en particular, pues ello implica esclarecer y completar los datos acerca de las actividades del pasado.

Tanto la definición como la caracterización de las actividades pasadas, no han sido un tópico desarrollado en la literatura $\mathrm{O}$ estudios bioantropológicos y bioarqueológicos. Aun así, los MOA siguen siendo utilizados para profundizar en la reconstrucción de actividades de las poblaciones pasadas, reconociendo su multifactorialidad (Jurmain et al. 2012) mediante la interpretación de diferencias o similitudes en los patrones de actividades, tanto a nivel inter-grupal como a nivel intra-grupal (Hawkey y Merbs 1995; Santana 2009-2010).

Por otra parte, algunos elementos claves para establecer factores ambientales y biomecánicos a los que encuentra expuesto el sistema locomotor en la ejecución de la actividad son: los gestos técnicos utilizados en la cadena operativa ${ }^{1}$, entendiéndose esta como una categoría de análisis que permite visualizar un esquema de acción coherente y secuencial que tiene por objetivo la transformación material por medio de la conjunción de elementos físicos, teóricos y prácticos (Salinas y Salazar 2008). Si se quiere caracterizar y definir una actividad, se debe comprender la producción del movimiento, integrando en su estudio factores socioculturales y fisiológicos, debido a que los gestos técnicos realizados

${ }^{1}$ Concepto de Chaine Operatorie, desarrollado por Leroi-Gourhan (1964) no son azarosos, son formas de hacer, que dependen de componentes sociales, históricos y ambientales que inciden en la forma de ejecutarlo (Ingold 2000; Mauss 1934). De manera similar, el sistema nervioso (SN) en cada nueva postura o movimiento registra un esquema plástico de las sensaciones suscitadas por la nueva postura y adapta la ejecución del movimiento, evitando la incomodidad postural y fatiga, contrarrestando la gravedad y cargas mecánicas a las que se encuentra sometido el sistema locomotor (Appendino et al. 2002).

A partir de lo expuesto, creemos que realizar e integrar estudios experimentales cinemáticos de los gestos técnicos permitirían reducir algunos errores de interpretación en la reconstrucción de actividades pasadas, siempre que se tenga en cuenta que: (a) detrás del uso o creación de los objetos, existe una lógica de hacer las cosas, por lo tanto existe una asociación entre las tareas y los objetos utilizados para realizarlas, reflejándose en los instrumentos la manera de ser usados (Bracco et al. 1991; D'Errico 1992); (b) los objetos se usan mediante una técnica, entendiéndose ésta como un conjunto de destrezas y conocimientos a nivel conductual y cognitivo individual que permite realizar una acción transformadora de forma intencional y reiterativa (Ingold 2000); (c) a nivel grupal existen gestos cotidianos similares y repetitivos que son adoptados por grupos en particular, sobre todo en las actividades de trabajo (Mauss 1934); (d) la postura es provocada y modificada por el pensamiento y la naturaleza interna de cada individuo, pero a su vez las adaptaciones posturales son resultado de la retroalimentación sensorial con el medio (Appendino et al. 2002); (e) a nivel individual no siempre se emplea una estrategia similar de movimiento para llevar a cabo una tarea en particular o una misma persona puede emplear diferentes estrategias al realizar la misma tarea (Bracco et al. 1992); y (f) el músculo esquelético, en condiciones normales funciona de forma voluntaria bajo el control del SNC, la graduación de la contracción muscular radica en un aumento de la frecuencia de descarga en cada unidad motora (UM) y en el reclutamiento de ésta (Maulén 2005), la electromiografía (EMG) detecta la activación de éstas, mediante una señal que representa la suma de potenciales de acción de las UMs (Fernández et al. 2007), por lo tanto, las demandas de activación de contracción muscular de un movimiento pueden ser observadas por medio de la EMG.

Bajo esta perspectiva, esta investigación es un estudio experimental cinemático que considera como antecedentes los resultados previos de investigaciones arqueológicas de actividades de extracción minera prehispánica en el Norte de Chile. Los objetivos de este estudio son: comparar la variabilidad de la activación eléctrica muscular de once músculos superficiales del miembro superior durante la ejecución de tres gestos técnicos empleados en las tareas de extracción y 
procesamiento de minerales de un contexto prehispánico de minería; y analizar diferencias de activación eléctrica muscular entre un mismo gesto técnico a nivel de extremidad superior adoptando diferentes posturas de tronco y extremidades inferiores.

\section{Antecedentes de las actividades mineras de Taltal}

La extracción y el procesamiento de pigmentos minerales tanto en la prehistoria mundial como en nuestro continente constituyó una actividad sistemática (Salazar et al. 2011), observándose en el Norte de Chile múltiples evidencias de minería prehispánica (Blanco et al. 2017; Salazar y Vilches 2014). Para este estudio se utilizarán los materiales provenientes de la Mina San Ramón 15 ( $\left.25^{\circ} 23^{\prime} 02^{\prime \prime} \mathrm{S} / 70^{\circ} 26^{\prime} 30^{\prime \prime} \mathrm{W}\right)$, ubicada en la cordillera de la costa de la región de Antofagasta, $5 \mathrm{Km}$. al Noreste de la localidad de Taltal, siendo el sitio con evidencia de actividad minera más antiguo en América, explotado inicialmente en el Período Arcaico temprano (10,0008,200 cal. A.C.) y posteriormente durante el período Arcaico Tardío (2,300-1,900 cal. A.C.) (Salazar et al. 2013). Si bien se postula que no existió una variación en cuanto a las herramientas utilizadas en ambos periodos, se ha planteado un cambio en la estrategia de extracción del mineral, pasando de un sistema que profundizaba la búsqueda de la veta a uno que se ampliaba hacia lateral (Salazar et al. 2011; Salinas et al. 2012).

El conjunto instrumental identificado para la explotación del yacimiento consiste principalmente de instrumentos macrolíticos (percutores, martillos, yunques y lascas desprendidas de éstos), registrándose cerca de mil martillos líticos, de los cuales el $99 \%$ se caracteriza por la ausencia de un sistema de enmangue (Salazar et al. 2011; Salazar et al. 2013; Salinas et al. 2012), lo cual marca una diferencia fundamental con las herramientas utilizadas en la extracción minera de Chile en tiempos históricos (Millán 2004). Finalmente, el conjunto artefactual arqueológico se complementa con la presencia de herramientas elaboradas sobre material malacológico y algunos artefactos de óseo animal (Salazar et al. 2011; Salazar et al. 2013).

\section{Metodología}

\section{Muestra}

La muestra utilizada para la evaluación cinemática fue de 16 individuos de sexo masculino, edad promedio 22 \pm 3 años, con características antropométricas similares (Estatura $=1,67 \pm 5 \mathrm{~cm}$.; Peso $=72 \pm 4 \mathrm{Kg}$ ). Siendo un $56,2 \%$ diestro y $43,8 \%$ zurdos. Para la selección de voluntarios se establecieron como elementos excluyentes la realización de actividad física de forma recurrente, y la presencia de traumatismo y/o lesiones actuales o pasadas en el sistema locomotor y/o asociados. Esto último, con el fin de minimizar el riesgo de adecuar el movimiento de acuerdo a algún patrón pre-establecido producto de la realización de algún gesto técnico previamente aprendido, o alguna restricción en el movimiento producto de un traumatismo $u$ otro factor patológico. Cabe mencionar que la exclusión de voluntarios de sexo femenino en estudio fue por criterios metodológicos, pues el porcentaje de tejido adiposo es mayor sobre algunas áreas de medición (e.g pectoral mayor) e interferiría en la lectura electromiográfica. Los voluntarios firmaron un consentimiento informado previamente a la experimentación.

\section{Gestos Técnicos Replicados}

Los posibles gestos técnicos y los instrumentos que se utilizaron en este estudio fueron seleccionados a partir del análisis de las huellas de uso de los instrumentos extractivos encontrados en el contexto de la Mina San Ramón 15 (en adelante SR-15), integrando además para su interpretación actividades de arqueología experimental y antecedentes etnográficos (véase Parra 2014; Salinas et al. 2012;). En este trabajo se presentan los datos obtenidos de la ejecución de tres posibles gestos técnicos más representativos para el miembro superior, de la actividad de extracción y procesamiento de pigmentos minerales. Además, se considera variantes en diferentes posturas de tronco y piernas, manteniendo el mismo gesto técnico en miembro superior. Los gestos técnicos corresponden a: 1) sujeto sentado sobre sus rodillas con las piernas flexionadas hacia posterior, quedando la zona glútea sobre la zona sural, 2) en posición de cuclillas, 3) de pie y 4) sentado en el suelo con las piernas extendidas anteriormente y abiertas en posición de $\mathrm{V}$.

Gesto Técnico I. La mano dominante sujeta longitudinalmente un instrumento -guijarro- cilíndrico de piedra (medidas: largo $15 \mathrm{~cm}$, diámetros $12 \mathrm{~cm}$. y 8 $\mathrm{cm}$.; peso 1,2 Kg.), la extremidad superior dominante ejecuta un golpe de superior a inferior como si se estuviera percutiendo. Se realiza una flexión/extensión de antebrazo, además el movimiento se ve acompañado de una rotación interna y flexión del brazo, flexión de la columna vertebral. El antebrazo se mantiene en posición neutra de prono-supinación en el movimiento, mientras sujeta el instrumento. El gesto técnico se realiza en todas las variantes mencionadas.

Gesto Técnico II. Ambas manos sujetan el borde lateral longitudinalmente un instrumento -guijarroparalelepípedo de piedra (medidas: Largo $20 \mathrm{~cm}$., ancho $15 \mathrm{~cm}$. y $8 \mathrm{~cm}$.; peso 3,2 Kg.). El gesto se realiza utilizando las dos extremidades superiores, ejecutando un golpe de superior a inferior como si se estuviera percutiendo a dos manos. Este movimiento se realiza mediante una flexión/extensión de antebrazo, pero además se ve acompañado por la rotación interna en una extremidad superior y una ligera rotación externa 
en la extremidad contra lateral. El movimiento incluye una flexión de columna vertebral, los antebrazos se mantienen en posición neutra de prono-supinación mientras ambas manos sujetan el instrumento. En la posición inicial uno de los hombros está ligeramente más a superior en aducción. El gesto técnico se realiza en todas las variantes mencionadas.

Gesto Técnico III. La mano dominante sujeta por la cara superior un instrumento -guijarro-cilíndrico de piedra (medidas: largo $15 \mathrm{~cm}$, diámetros $12 \mathrm{~cm}$.y $8 \mathrm{~cm}$.; peso 1,2 Kg.). El gesto se ejecuta utilizando la extremidad superior dominante, se mueve el brazo y antebrazo en dirección de anterior/posterior, como si se estuviera moliendo sobre una superficie. El movimiento considera una ligera flexión de brazo, flexión/extensión de antebrazo, flexión de la columna vertebral, los antebrazos se mantienen en pronación mientras se ejecuta el movimiento. El gesto técnico se realiza en las variantes 1 y 2 .

\section{Variables Observadas}

Se midió la activación eléctrica de los músculos: deltoides porción anterior (mDA), deltoides porción media (mDM), deltoides porción posterior (mDP), trapecio porción superior (mTS), serrato anterior (mSA), pectoral mayor porción horizontal (mPMH), pectoral mayor porción oblicua (mPMO), bíceps braquial (mBB), tríceps braquial (mTB), flexor radial (mFR) y extensor radial (mER).

\section{Procedimientos}

El registro de los datos fue realizado en el Laboratorio de Kinesiología Aplicada de la Universidad Católica de la Santísima Concepción. Para estandarizar la realización de cada gesto, se entregaron las mismas indicaciones a los sujetos, utilizando como ejemplo visual una grabación de lo movimientos donde se ejemplificaba el uso de las herramientas arqueológicas A cada sujeto se adosaron 14 marcadores reflectantes esféricos, de $10 \mathrm{~mm}$. de diámetro en el tronco y su miembro superior dominante. Además, se ubicaron 11 sensores inalámbricos de electromiografía de superficie (sEMG, marca DELSYS, modelo TRIGNO), uno en cada vientre muscular de los músculos previamente descritos según las normas descritas por la SENIAM.

El procedimiento comenzó con el registro de la actividad muscular de reposo para cada músculo en posición sedente con el miembro superior dominante ubicada en forma relajada sobre una camilla. Posteriormente, el sujeto realizó una serie de 10 movimientos de percusión o molienda, sobre una pieza soporte de madera de dimensiones $20 \times 20 \times 40 \mathrm{~cm}$, sujeta firmemente sobre una base metálica o apoyada en el suelo, dependiendo el gesto a replicar. Los 10 movimientos corresponden a los gestos técnicos y variantes ya descritos.

En cada movimiento, se les solicitó a los sujetos que tomaran el instrumento con su mano dominante (gesto I y III) y ambas manos (gesto II) y que realizaran cinco ciclos de percusión o molienda, impactando la pieza soporte "como si quisiera extraer o moler el mineral", se considera que cada ciclo comienza y termina en el momento en que el instrumento golpea o contacta la pieza soporte. Durante cada gesto se registró la ubicación espacial de los marcadores reflectantes mediante un sistema de seis cámaras infrarrojas (marca VICON, modelo BONITA 10), a una frecuencia de muestreo de $100 \mathrm{~Hz}$. También durante cada intento se registró la actividad eléctrica muscular mediante los sensores de sEMG, a una frecuencia de $2000 \mathrm{~Hz}$. Ambos registros fueron integrados y sincronizados mediante el módulo MX GIGANET, de VICON.

El estudio consideró la medición del nivel de actividad eléctrica muscular, posición angular y velocidad angular, pero en este trabajo solo se presentará las de actividad muscular. A partir de los datos registrados se analizaron únicamente el segundo, el tercer y el cuarto ciclo de cada gesto, completando un total de 30 ciclos por sujeto. La señal de sEMG de cada músculo fue procesada con el software NEXUS de VICON. Primero se aplicó un filtro Butterworth de $4^{\circ}$ orden en cada señal, para luego ser rectificada para calcular la raíz media cuadrática (RMS), con una ventana de $100 \mathrm{~ms}$, con el objeto de suavizar la señal. Luego, se promediaron las señales de los tres ciclos de cada gesto, para cada uno de los músculos. Posterior a eso se normalizó la magnitud de actividad muscular como el porcentaje de activación, considerando el $0 \%$ como la actividad registrada durante el reposo y el $100 \%$, como la máxima actividad registrada para cada músculo en cualquiera de los 10 movimientos. Por último, la magnitud utilizada de activación de cada músculo es el promedio del segundo, tercer y cuarto ciclo de cada gesto estudiado.

\section{Análisis estadístico}

En cada gesto técnico estudiado, es decir los tres movimientos y sus variantes, se procedió, mediante el paquete de análisis descriptivo del programa estadístico IBM SPSS.20, a calcular la media aritmética $(\bar{X})$, la desviación estándar (DS) y coeficiente de variación (\%CV), para observar la dispersión de los datos, se excluyeron aquellos valores atípicos que podían incidir en el sesgo de los resultados. Además, se aplicó la prueba $\mathrm{H}$ de Kruskal-Wallis para comparar los porcentajes de activación muscular entre las diferentes posturas de un mismo gesto técnico.

\section{Resultados}

1. Variabilidad del porcentaje de activación muscular por movimiento-entre sujetos

En la tabla 1 se observan los promedios $(\bar{X})$ de los porcentajes de activación muscular (\%AC), su desviación 
estándar (DS), coeficiente de variación (\%CV) y Rangos. En ésta se refleja la dispersión de los datos y se evidencia una alta variabilidad de activación muscular, entre sujetos, durante la ejecución de un mismo movimiento.

Gesto I: Se observa en todas las posturas de este gesto una mayor dispersión de \%AC en los músculos mTS, mSA, mPH, mPO, mFR. En las posturas De Pie y Piernas $V$, mDA y mBB aumentan su dispersión, de igual manera el mDP en las posturas de Cuclillas y Piernas V. El mDM incrementa en la postura De Pie y mTB en las Piernas V.

Gesto II: Se observa en todas las posturas de este gesto una mayor dispersión de \%AC en los músculos mSA, mPH, mPO, Mtb, mTS, éste último a excepción de la posición de cunclilla. En las posturas De Pie y Piernas $V$, mDM y mFR aumentan su dispersión, de igual manera el mDP en la postura De Pie y mBB en las Piernas V.

Gesto III: Se observa en todas las posturas, una dispersión sobre $50 \%$ sobre la media de todos los músculos, a excepción de la posición del mDM en la postura Cuclillas y del mTS en la postura Rodillas. Siendo este gesto el con mayor variabilidad muscular.
Cabe destacar el mSA presenta una alta dispersión en todos los movimientos, al contrario del mER.

\section{Comparación del porcentaje de activación muscular entre posturas adoptadas para un mismo gesto técnico}

Se presentan los resultados de la prueba Kruskal-Wallis en la tabla 2 con el fin de comparar la variabilidad del \%AM con el mismo gesto técnico, pero con diferente postura de tronco y piernas.

Gesto técnico I: La distribución de las medias del gesto técnico I se presentan en la figura 1, el patrón de actividad muscular es más elevado en los músculos que participan en la flexión/extensión, elevación y rotación del hombro en la postura de cuclillas. Al comparar los rangos medios de las diferentes posturas se observan diferencias estadísticamente significativas únicamente en el mSA $(p=0,042)$. Sin embargo, al cotejar de forma pareada la postura de rodillas y cuclillas, existe una diferencia significativa entre los porcentajes de activación muscular en mDM $(p=0,02)$, siendo mayores los grados de activación en la postura de cuclillas. Lo mismo sucede al comparar la postura de cuclillas con

\begin{tabular}{|c|c|c|c|c|c|c|c|c|c|c|c|c|c|}
\hline \multicolumn{3}{|c|}{ MOVIMIENTOS } & \multicolumn{11}{|c|}{ MUSCULOS } \\
\hline GESTO & POSTL & JRA & DA & DM & DP & TS & SA & PH & PO & BB & TB & $F R$ & ER \\
\hline \multirow{16}{*}{$\begin{array}{c}\text { GESTO TÉCNICO } 1 \\
\text { Golpe } \\
\text { Superior-Inferior } \\
\text { Utilizando una mano }\end{array}$} & \multirow{4}{*}{ Rodillas } & B & 9,0 & 15,8 & 8,6 & 9,3 & 28,2 & 7,2 & 9,6 & 12,1 & 15,9 & 12,3 & 36,1 \\
\hline & & DS & 3,4 & 6,0 & 3,2 & 5,6 & 16,6 & 3,7 & 5,7 & 5,0 & 6,5 & 6,2 & 9,6 \\
\hline & & $\% C V$ & 37,8 & 38,0 & 37,2 & 60,2 & 58,9 & 51,4 & 59,4 & 41,3 & 40,9 & 50,4 & 26,6 \\
\hline & & Rango & 13,0 & 22,7 & 11,4 & 18,1 & 59,8 & 12,6 & 18,4 & 17,7 & 23,6 & 20,1 & 33,1 \\
\hline & \multirow{4}{*}{ Cuclillas } & B & 11,1 & 21,4 & 9,8 & 12,7 & 33,0 & 7,8 & 8,8 & 13,6 & 15,7 & 10,8 & 34,3 \\
\hline & & DS & 5,6 & 7,1 & 7,1 & 8,4 & 22,8 & 3,9 & 6,4 & 4,5 & 5,6 & 6,4 & 8,9 \\
\hline & & $\% \mathrm{CV}$ & 50,5 & 33,2 & 72,4 & 66,1 & 69,1 & 50,0 & 72,7 & 33,1 & 35,7 & 59,3 & 25,9 \\
\hline & & Rango & 19,7 & 26,2 & 25,9 & 28,4 & 84,0 & 13,2 & 19,5 & 17,2 & 18,5 & 23,0 & 28,3 \\
\hline & \multirow{4}{*}{ De Pie } & B & 8,3 & 15,8 & 20,3 & 11,4 & 20,1 & 8,1 & 9,7 & 15,4 & 16,7 & 17,6 & 31,9 \\
\hline & & DS & 5,1 & 8,3 & 17,9 & 8,0 & 22,7 & 4,3 & 8,8 & 7,9 & 5,6 & 10,8 & 16,9 \\
\hline & & $\% C V$ & 61,4 & 52,5 & 88,2 & 70,2 & 113 & 53,1 & 90,7 & 51,3 & 33,5 & 61,4 & 53,0 \\
\hline & & Rango & 20,5 & 29,4 & 53,7 & 28,1 & 73,5 & 15,2 & 30,2 & 25,2 & 20,3 & 31,8 & 61,0 \\
\hline & \multirow{4}{*}{ Piernas V } & B & 9,8 & 14,7 & 8,1 & 14,5 & 16,1 & 9,6 & 11,3 & 10,1 & 13,2 & 10,8 & 28,4 \\
\hline & & DS & 5,4 & 6,8 & 3,7 & 9,1 & 18,0 & 6,1 & 7,5 & 5,4 & 7,4 & 6,1 & 7,6 \\
\hline & & $\% \mathrm{CV}$ & 55,1 & 46,3 & 45,7 & 62,8 & 112 & 63,5 & 66,4 & 53,5 & 56,1 & 56,5 & 26,8 \\
\hline & & Rango & 21,5 & 27,0 & 13,9 & 27,5 & 63,4 & 20,5 & 22,0 & 18,4 & 25,2 & 2 & 24, \\
\hline \multirow{16}{*}{$\begin{array}{l}\text { GESTO TÉCNICO } 2 \\
\text { Golpe } \\
\text { Superior-Inferior } \\
\text { Utilizando dos mano }\end{array}$} & \multirow{4}{*}{ Rodillas } & B & 31,4 & 29,0 & 7,9 & 19,5 & 45,5 & 27,9 & 30,0 & 34,4 & 19,5 & 27,5 & 41 \\
\hline & & DS & 5,9 & 11,5 & 4,0 & 12,9 & 18,9 & 18,3 & 16,0 & 8,5 & 10,8 & 12,4 & 13, \\
\hline & & $\% \mathrm{CV}$ & 18,8 & 39,7 & 50,6 & 66,2 & 41,5 & 65,6 & 53,3 & 24,7 & 55,4 & 45,1 & 32,7 \\
\hline & & Rango & 20,2 & 32,7 & 13,4 & 40,4 & 62,2 & 51,4 & 48,9 & 27,8 & 27,0 & 38,5 & 41,9 \\
\hline & \multirow{4}{*}{ Cuclillas } & B & 37,2 & 36,2 & 11,1 & 39,4 & 45,4 & 36,9 & 26,4 & 33,1 & 16,7 & 22,8 & 40,9 \\
\hline & & DS & 12,2 & 10,4 & 5,0 & 12,9 & 22,6 & 19,2 & 17,5 & 11,8 & 8,5 & 9,7 & 9,5 \\
\hline & & $\% \mathrm{CV}$ & 32,8 & 28,7 & 45,0 & 32,7 & 49,8 & 52,0 & 66,3 & 35,6 & 50,9 & 42,5 & 23, \\
\hline & & Rango & 36,0 & 36,0 & 16,7 & 49,3 & 66,7 & 56,7 & 54,7 & 38,0 & 27,5 & 35,0 & 30,0 \\
\hline & \multirow{4}{*}{ De Pie } & B & 28,8 & 23,0 & 10,8 & 20,9 & 28,4 & 28,8 & 30,0 & 27,7 & 16,0 & 26,7 & 35 \\
\hline & & DS & 9,0 & 12,3 & 6,0 & 11,0 & 22,9 & 14,6 & 15,6 & 12,2 & 8,5 & 13,8 & 11 \\
\hline & & $\% \mathrm{CV}$ & 31,3 & 53,5 & 55,6 & 52,6 & 80,6 & 50,7 & 52,0 & 44,0 & 53,1 & 51,7 & 31, \\
\hline & & Rango & 27,6 & 40,0 & 21,7 & 38,3 & 75,5 & 46,7 & 48,2 & 42,6 & 28,0 & 46,7 & 43,3 \\
\hline & \multirow{4}{*}{ Piernas V } & B & 26,9 & 24,3 & 7,7 & 28,7 & 29,2 & 30,7 & 27,1 & 23,1 & 12,8 & 19,4 & 33,8 \\
\hline & & DS & 5,2 & 12,9 & 3,4 & 16,1 & 19,4 & 16,6 & 15,1 & 13,1 & 7,4 & 10,4 & 8,7 \\
\hline & & $\% \mathrm{CV}$ & 19,3 & 53,1 & 44,2 & 56,1 & 66,4 & 54,1 & 55,7 & 56,7 & 57,8 & 53,6 & 25,7 \\
\hline & & Rango & 17,5 & 43,2 & 12,8 & 53,6 & 72,0 & 48,0 & 48,1 & 41,9 & 25,1 & 38,6 & 33,1 \\
\hline \multirow{8}{*}{$\begin{array}{l}\text { GESTO TÉCNICO } 3 \\
\text { Molienda } \\
\text { Anterior- Posterior } \\
\text { Utilizando una mano }\end{array}$} & \multirow{4}{*}{ Rodillas } & P & 8,3 & 20,1 & 22,8 & 5,2 & 21,9 & 7,2 & 14,8 & 51 & 27 & 28,4 & 36 \\
\hline & & DS & 4,6 & 9,0 & 13,0 & 1,7 & 18,8 & 5,5 & 11,9 & 4,9 & 14,2 & 15,0 & 19, \\
\hline & & $\% C V$ & 55,4 & 44,8 & 57,0 & 32,7 & 85,8 & 76,4 & 80,4 & 96,1 & 51,8 & 52,8 & 52,8 \\
\hline & & Rango & 14,6 & 28,8 & 44,3 & 7,0 & 69,6 & 19,0 & 45,0 & 15,8 & 48,3 & 48,7 & 61,5 \\
\hline & \multirow{4}{*}{ Cuclillas } & B & 8,0 & 21,9 & 23,8 & 10,0 & 24,8 & 8,0 & 20,8 & 4,0 & 29,7 & 31,2 & 30 \\
\hline & & DS & 5,2 & 6,0 & 16,5 & 7,0 & 19,4 & 5,6 & 16,1 & 3,0 & 15,4 & 22,8 & 24,2 \\
\hline & & $\% \mathrm{CV}$ & 65,0 & 27,4 & 69,3 & 70,0 & 78,2 & 70,0 & 77,4 & 75,0 & 51,9 & 73,1 & 80, \\
\hline & & Rangc & 16,6 & 20,7 & 59,4 & 24,7 & 65,7 & 19,9 & 48,9 & 11,6 & 50,9 & 80,0 & 83,9 \\
\hline
\end{tabular}

la de pie para el mER $(p=0,03)$. Al contrastar las posturas cuclillas con sentado en piernas $V$, se observan mayores grados en la última, para los mSA $(p=0,007), \operatorname{mDM}(p=0,023)$ y $\operatorname{mER}(p=0,036)$. Cabe señalar que en los dos últimos músculos mencionados, las diferencias entre los porcentajes de activación muscular al comparar las cuatro posturas no son significativas, dicha homogeneidad de valores puede ser debido a que en ambos casos existe un elevado grado de variabilidad que al conjugar y comparar las

Tabla 1. Resumen de Media Aritmética $(\bar{X})$, Desviación Estándar (DS), Coeficiente de Variación $(\% \mathrm{CV})$ y Rango. DA: Deltoides (Porción Anterior); DM: Deltoides (Porción Media); DP: Deltoides (Porción Posterior) TS: Trapecio (Porción Superior); SA: Serrato Anterior; PH: Pectoral Mayor (Porción Horizontal); PO: Pectoral Mayor (Porción Oblicua); BB: Bíceps Braquial; TB: Tríceps Braquial; FR: Flexor Radial; ER: Extensor Radial

Table 1. Arithmetic average $(\bar{X})$, Standard deviation (SD), Variability Coefficient summary (\%VVC) and Range DA: Deltoid (Anterior Portion); DM: Deltoid (Medial portion); DP: Deltoid (Posterior portion) TS: Trapeze (Superior portion); SA: Anterior Serratus; PH: Mayor pectoral (Horizontal portion); PO: Mayor pectoral (Oblique portion); BB: Brachial Biceps; BT: Brachial Triceps; RF: Radial Flexor; ER: Radial Extensor. 


\begin{tabular}{|c|c|c|c|c|c|c|c|c|c|c|c|c|c|}
\hline \multicolumn{3}{|c|}{ MOVIMIENTOS } & \multicolumn{11}{|c|}{ MúSCULOS } \\
\hline GESTO & POSTURA & & DA & DM & DP & TS & SA & PH & PO & BB & TB & FR & ER \\
\hline \multirow{5}{*}{$\begin{array}{c}\text { GESTO TÉCNICO } 1 \\
\text { Golpe } \\
\text { Superior-Inferior } \\
\text { Utilizando una mano }\end{array}$} & Rodillas & $R$ & 27,9 & 26,8 & 27,7 & 24,3 & 22,3 & 22,5 & 25,0 & 27,3 & 30,3 & 29,7 & 34,6 \\
\hline & Cuclillas & $R$ & 37,1 & 40,9 & 29,9 & 33,9 & 26,7 & 26,5 & 26,3 & 34,2 & 32,5 & 28,6 & 34,5 \\
\hline & De Pie & $R$ & 26,5 & 21,9 & 37,5 & 29,7 & 15,7 & 24,1 & 25,0 & 36,3 & 33,3 & 37,7 & 31,0 \\
\hline & Pierna & $\mathrm{R}$ & 31,1 & 26,3 & 27,6 & 34,8 & 13,4 & 29,0 & 31,9 & 24,6 & 24,2 & 25,9 & 21,9 \\
\hline & Grupal & $p$ & ,374 & ,084 & ,335 & ,327 & ,042 & ,681 & ,618 & 208 & ,463 & 290 & 151 \\
\hline \multirow{5}{*}{$\begin{array}{c}\text { GESTO TÉCNICO } 2 \\
\text { Golpe } \\
\text { Superior-Inferior } \\
\text { Utilizando dos mano }\end{array}$} & Rodillas & $R$ & 30,6 & 28,1 & 26,4 & 21,9 & 19,8 & 22,0 & 25,6 & 35,0 & 35,0 & 32,1 & 36,5 \\
\hline & Cuclillas & $\mathrm{R}$ & 38,5 & 40,4 & 31,4 & 41,0 & 24,5 & 27,2 & 25,0 & 34,9 & 29,0 & 29,7 & 33,0 \\
\hline & De Pie & $R$ & 25,6 & 23,8 & 34,0 & 23,5 & 13,6 & 21,7 & 28,4 & 26,8 & 30,7 & 31,5 & 27,4 \\
\hline & Piernas V & $R$ & 24,3 & 26,7 & 25,6 & 31,8 & 15,6 & 23,3 & 24,8 & 22,6 & 24,0 & 25,0 & 22,5 \\
\hline & Grupal & $p$ & ,099 &, 042 &, 464 & ,009 & 121 & ,755 & ,918 & , 125 & ,379 & ,657 & 131 \\
\hline \multirow{3}{*}{$\begin{array}{l}\text { GESTO TÉCNICO } 3 \\
\text { Molienda } \\
\text { Anterior- Posterior } \\
\text { Utilizando una mano }\end{array}$} & Rodillas & $R$ & 15,7 & 14,0 & 15,2 & 12,8 & 10,3 & 11,5 & 12,9 & 15,6 & 14,9 & 15,4 & 17,2 \\
\hline & Cuclillas & $R$ & 16,3 & 18,3 & 16,8 & 19,4 & 10,7 & 14,6 & 15,2 & 16,4 & 17,1 & 16,6 & 14,8 \\
\hline & Grupal & $p$ & ,843 & ,206 & ,635 &, 044 & ,880 & ,301 & ,467 & ,797 &, 502 & ,722 & ,453 \\
\hline
\end{tabular}

Tabla 2. Prueba Kruskal-Wallis Resumen de rangos (R) y significancia ( $p)$ DA: Deltoides (Porción Anterior); DM: Deltoides (Porción Media); DP: Deltoides (Porción Posterior) TS: Trapecio (Porción Superior); SA: Serrato Anterior; PH: Pectoral Mayor (Porción Horizontal); PO: Pectoral Mayor (Porción Oblicua); BB: Bíceps Braquial; TB: Tríceps Braquial; FR: Flexor Radial; ER: Extensor Radial

Table 2. Kruskal-Wallis Test, Range Summary and p-value. DA: Deltoid (Anterior Portion); DM: Deltoid (Medial portion); DP: Deltoid (Posterior portion) TS: Trapezius (Superior portion); SA: Anterior Serratus; PH: Pectoralis Major (Horizontal portion); PO: Pectoralis Major (Oblique portion); BB: Biceps Brachii; BT: Triceps Brachii; RF: Flexor Carpi Radialis; ER: Extensor Carpii Radialis. cuatro muestras podría matizar los valores extremos y las diferencias.

La mayor activación muscular del mDM y mSA en posición de cuclillas se podría deber a que es más complejo auxiliar el movimiento con la flexión del tronco y las fibras musculares de ambos músculos deben abducir y sostener los movimientos del brazo que implican elevación y rotación de la escápula (Peterson et al. 2007) sin ayuda del tronco. En el caso de mER, se activa en mayor grado en las posiciones que el tronco se encuentra más cerca del suelo y la distancia de la mano al soporte es menor, lo anterior podría ser producto en que en dichas posturas la mano recibe la fuerza del impacto del golpe del instrumento

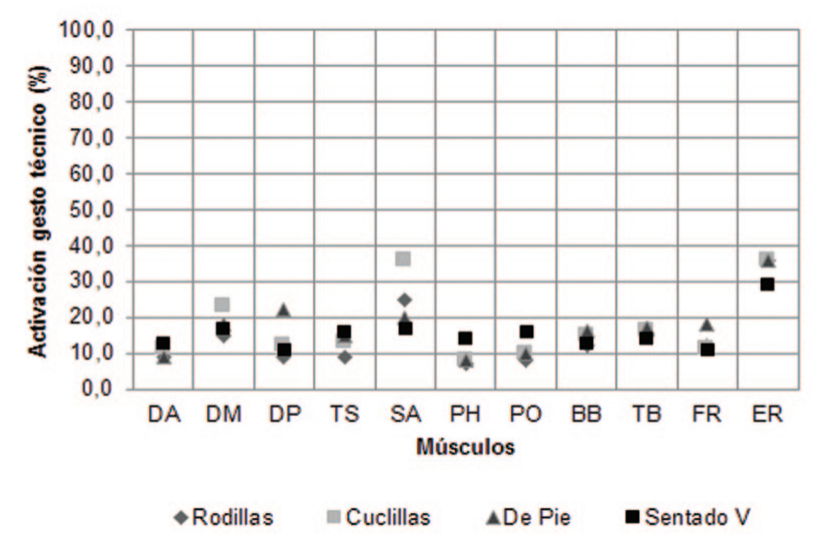

Figura 1. Resumen de los promedios $(\bar{X})$ de activación muscular del gesto técnico I, en todas sus posturas. DA: Deltoides (Porción Anterior); DM: Deltoides (Porción Media); DP: Deltoides (Porción Posterior) TS: Trapecio (Porción Superior); SA: Serrato Anterior; PH: Pectoral Mayor (Porción Horizontal); PO: Pectoral Mayor (Porción Oblicua); BB: Bíceps Braquial; TB: Tríceps Braquial; FR: Flexor Radial; ER: Extensor Radial

Figure 1. Arithmetic average $(\overline{\mathrm{X}})$ of muscle activation technical gesture I Summary. DA: Deltoid (Anterior Portion); DM: Deltoid (Medial portion); DP: Deltoid (Posterior portion) TS: Trapezius (Superior portion); SA: Anterior Serratus; PH: Pectoralis Major (Horizontal portion); PO: Pectoralis Major (Oblique portion); BB: Biceps Brachii; BT: Triceps Brachii; RF: Flexor Carpi Radialis; ER: Extensor Carpii Radialis en el suelo y debe mantenerlo firmemente agarrado para seguir ejecutando el movimiento (Peterson et al. 2007).

Gesto técnico II: La distribución de las medias del gesto técnico II se presenta en la figura 2, el patrón de actividad muscular es más elevado en los músculos que participan en la flexión/extensión, elevación y rotación del hombro en la postura de cuclillas. Al comparar los rangos medios de las diferentes posturas se observan diferencias estadísticamente significativas para el mDM $(p=0,042)$ y $\mathrm{mTS}(\mathrm{p}=0,009)$ (ver figura 2$)$. Cuando dicha comparación de posturas se realiza de forma pareada, las diferencias entre los porcentajes de activación muscular siguen evidenciándose entre los mDM $(p=0,037)$ y mTS $(p=0,004)$ siendo mayores los grados de activación en la postura de cuclillas respecto a la de rodillas, lo que se repite al comparar la postura de cuclillas con la de pie $\operatorname{mDM}(p=0,01)$ y $m T S=0,05)$. En este caso en particular también se incrementa el grado del mDA $(P=0,22)$ y mSA $(p=0,04)$. Si bien éstas últimas no se observan al comparar las cuatro posturas, esto se puede deber a que en ambos casos existe un alto grado de variabilidad que podría ser homogeneizado al tener un grupo mayor comparándose.

También existe una diferencia entre los grados de activación de $\operatorname{mDA}(p=0,042)$ y $\operatorname{mDM}(p=0,038)$ entre la postura de cuclillas y sentado en piernas abiertas en $\mathrm{V}$, siendo siempre más elevados en la primera postura. Esto nos lleva a pensar que probablemente en esta posición se requiera un mayor esfuerzo para ejecutar el gesto técnico, pues se debe mantener estable la columna y esta no participa de forma activa en movimiento. Tanto la activación mayor de mDM y mTS se podría deber a que en posición de cuclillas la flexión del tronco es más compleja y dichas fibras musculares participan en la abducción y sostienen durante los movimientos que implican elevación y rotación de la escápula (Peterson et al. 2007).

Gesto técnico III: La distribución de las medias del gesto técnico III se presenta en la figura 3, y el patrón de 


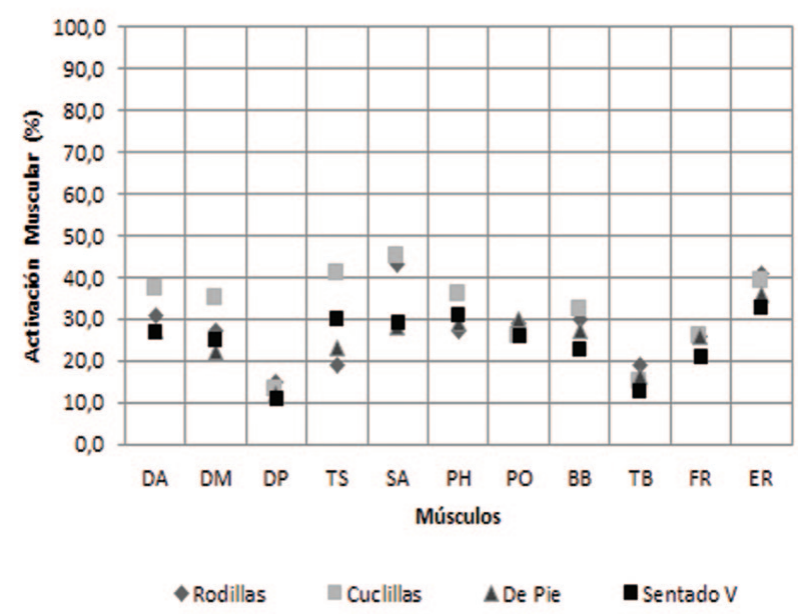

Figura 2. Resumen de los promedios $(\bar{X})$ de activación muscular del gesto técnico II, en todas sus posturas. DA: Deltoides (Porción Anterior); DM: Deltoides (Porción Media); DP: Deltoides (Porción Posterior) TS: Trapecio (Porción Superior); SA: Serrato Anterior; PH: Pectoral Mayor (Porción Horizontal); PO: Pectoral Mayor (Porción Oblicua); BB: Bíceps Braquial; TB: Tríceps Braquial; FR: Flexor Radial; ER: Extensor Radial

Figure 2. Arithmetic average $(\overline{\mathrm{X}})$ of muscle activation technical gesture II Summary. DA: Deltoid (Anterior Portion); DM: Deltoid (Medial portion); DP: Deltoid (Posterior portion) TS: Trapezius (Superior portion); SA: Anterior Serratus; PH: Pectoralis Major (Horizontal portion); PO: Pectoralis Major (Oblique portion); BB: Biceps Brachii; BT: Triceps Brachii; RF: Flexor Carpi Radialis; ER: Extensor Carpii Radialis.

actividad muscular es bastante similar, no se observan diferencias estadísticamente significativas entre las activaciones musculares de la postura de rodillas y en cuclillas, a excepción de mTS $(p=0,044)$, esta diferencia podría deberse a que en posición de cuclillas la flexión del tronco es más compleja y las fibras superiores de este músculo deben sostener la posición de elevación y rotación de la escápula (Peterson et al. 2007).

\section{Discusión}

Los resultados muestran que existe una alta variabilidad del \%AM entre sujetos al ejecutar un mismo movimiento, al menos la mitad de los músculos medidos presenta una dispersión superior el 50\% sobre la media grupal. Los músculos en los cuales se observa mayor variabilidad entre sujetos dependen del movimiento que se ejecute, es decir, si el gesto técnico tiene por objetivo acercarse a un objeto, son los músculos del hombro y aquellos que unen el tronco al brazo, los que presentan mayor variabilidad -mDA, mDP, mTS, $\mathrm{mPH}$. mPO-, todos estos músculos son partícipes ya sea de la rotación externa/ interna, abducción y aducción del hombro y brazo (Peterson et al. 2007).

Es interesante observar que la variabilidad de los músculos anteriormente mencionados, se incrementa en las posturas en cuales la zona inferior de la columna se encuentra más alejada del suelo, y en las cuales el sujeto puede flexionar de forma natural la columna vertebral para auxiliar el movimiento, durante el acercamiento de los brazos al soporte. Además, el movimiento puede ser ejecutado por el miembro superior con una mayor o menor rotación externa o interna o de forma más horizontal u oblicua. El mayor grado de dispersión del mDA y mFR en el gesto I en comparación al gesto II, puede ser explicado para el primero, en la posibilidad física de rotar y aducir el brazo con mayor libertad, lo que a su vez repercute en la cantidad de unidades motoras reclutadas y para el segundo, por las percepciones individuales, para controlar la sujeción del instrumento con una mano y la precisión del movimiento (Clarkson 2003).

En el caso del gesto III que tenía por objetivo moler pigmentos, en general todos los músculos incrementan su dispersión sobre la media, pero son los músculos del brazo, antebrazo y mano - $\mathrm{mDA}, \mathrm{mDP}, \mathrm{mPH}, \mathrm{mPO}, \mathrm{mBB}$, $m T B, m F R$ mER- que adquieren una alta dispersión en comparación a los otros gestos. De igual manera, los cuatro primeros participan rotación externa/interna, abducción y aducción del hombro y brazo (Peterson et al. 2007) y variabilidad dependa principalmente de la amplitud y forma del movimiento; en el caso de los cuatro últimos se debe principalmente a las funciones flexión/extensión de antebrazo y mano (Peterson et al. 2007).

Respecto a la comparación de posturas de cada gesto, es la acción de molienda la que presenta menores diferencias, posiblemente pues la acción no implica un

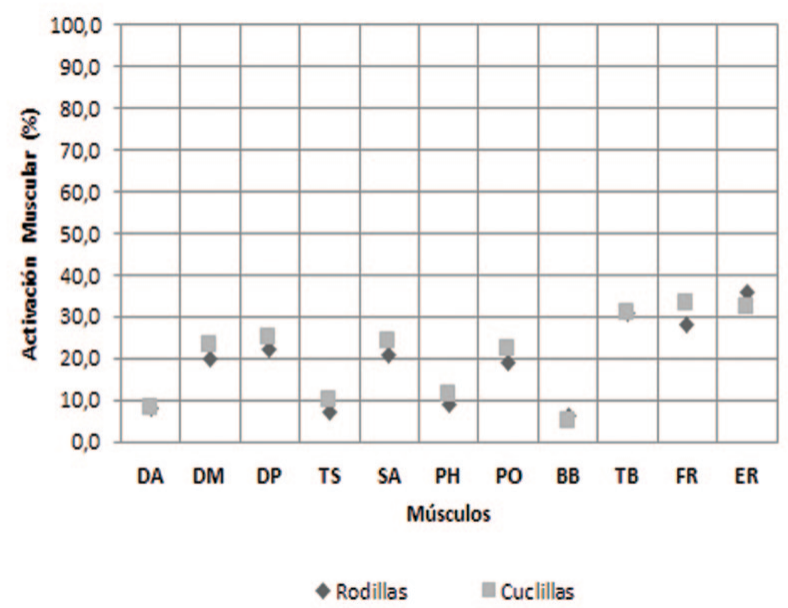

Figura 3. Resumen de los promedios $(\bar{X})$ de activación muscular del gesto técnico II, en todas sus posturas. DA: Deltoides (Porción Anterior); DM: Deltoides (Porción Media); DP: Deltoides (Porción Posterior) TS: Trapecio (Porción Superior); SA: Serrato Anterior; PH: Pectoral Mayor (Porción Horizontal); PO: Pectoral Mayor (Porción Oblicua); BB: Bíceps Braquial; TB: Tríceps Braquial; FR: Flexor Radial; ER: Extensor Radial

Figure 3. Arithmetic average $(\overline{\mathrm{X}})$ of muscle activation technical gesture II Summary. DA: Deltoid (Anterior Portion); DM: Deltoid (Medial portion); DP: Deltoid (Posterior portion) TS: Trapezius (Superior portion); SA: Anterior Serratus; PH: Pectoralis Major (Horizontal portion); PO: Pectoralis Major (Oblique portion); BB: Biceps Brachii; BT: Triceps Brachii; RF: Flexor Carpi Radialis; ER: Extensor Carpii Radialis. 
levantamiento de los hombros o un acercamiento de los segmentos del miembro superior hacia el objeto para ejecutar el gesto, en este caso el tronco no auxilia voluntariamente el movimiento, es decir, no se desplaza hacia adelante. En los gestos técnicos I y II (percusión) los músculos que actúan a nivel del hombro presentan mayores diferencias, el alto grado de variabilidad del gesto técnico II (percusión a dos manos) podría estar directamente relacionado con la distancia del miembro superior y el grado de acercamiento al objeto golpeado, es decir si el tronco del sujeto se flexiona acompañando al movimiento o se mantiene más cercano a la posición inicial, y/o las extremidades se levantan más en la ejecución del movimiento, por otra parte, en este gesto se utilizó un instrumento más grande y pesado, al aumentar el peso del objeto, las demandas biomecánicas a nivel fisiológicas aumentan), el desarrollo de la fuerza a nivel individual podría jugar un rol en la cantidad de unidades motoras activadas para poder generar un golpe más potente, pues aunque existen variaciones a nivel interindividual en los movimientos (Clarkson 2003, Hunter 2000) éstos responden a principios asociados a factores motores, tanto fisiológicos como ergonómicos que deben satisfacerse para realizar tareas específicas, por esta razón, a medida que el cuerpo adquiere la experiencia, las respuestas motoras tienden a ser más similares, pues se conoce las potencias y presiones que se deben aplicar (Pasciuto et al. 2014) y la precisión del golpe es mayor (Bril et al. 2010)

A partir de lo anterior, creemos que si se está trabajando en la interpretación de músculos, así como Niinimäki y colaboradores (2016) han evidenciado que se debe considerar la carga y tipo de ejercicio efectuado (resistencia o carga), creemos que también se debe evaluar las diferentes zonas de activación muscular, y observar en el registro óseo por área de inserción de acuerdo a las secciones de fibras y no de forma general, pensando que su activación depende de la amplitud del movimiento ejecutado, pudiendo actuar de forma conjunta o separada las fibras musculares. Por ejemplo, para el músculo deltoides, las fibras anteriores tienen funciones de flexión, las medias se activan mayormente para dar estabilidad al hombro, y las posteriores para la extensión del brazo (Peterson et al. 2007). De igual manera, las fibras del pectoral horizontal se activan durante los movimientos verticales de aducción y rotación interna, en cambio las fibras oblicuas lo hacen cuando el movimiento es de forma horizontal (Peterson et al. 2007).

Somos conscientes de que este tipo de estudios no correlaciona directamente el \%AM con un indicador óseo de actividad (Niinimäki et al. 2016), pero permite visualizar la importancia de considerar una perspectiva que considere las formas de hacer, en este caso considerando que el uso de instrumentos y saberes, fueron ejecutados por sujetos sociales y éstos desarrollaron sus habilidades y experticia dentro de un contexto social y ambiental en particular que pautó la forma de tomar decisiones, las tareas realizadas y técnicas utilizadas (Ingold 2002; Ingold 2008; Salazar y Salinas 2008). Por lo anterior, es que creemos que al analizar MOA, se debe no sólo considerar la dimensión biológica (e.g sexo, edad, factores hormonales, entre otros) y mecánica que influyen en el desarrollo de un indicador, sino las dimensiones ambientales y sociales que moldean la ejecución de una tarea y sus gestos técnicos.

Desde nuestro punto de vista y dado el carácter multivariado de la activación muscular, de debe mencionar que existen variadas limitantes metodológicas y analíticas en este estudio, por lo que recalcamos la naturaleza exploratoria de nuestro acercamiento al problema de investigación, creemos que estas limitantes podrían actuar de manera sinérgica y no excluyente. Una limitante corresponde al desconocimiento de la cadena productiva y las tareas específicas necesarias para realizar cada una de las acciones, siendo éstas muy necesarias para entender los requerimientos biomecánicos y poder generar una experimentación más controlada, entendiendo que las demandas de fuerza y la reclusión de unidades motoras del músculo eventualmente se saturan (Clarkson 2003; Maulén 2005). Diversos estudios del trabajo demuestran que no todas las partes del miembro superior reaccionan de la misma forma frente a un estímulo, la evidencia epidemiológica demuestra que dependiendo la zona del brazo estudiada, el factor de riesgo a producir "una lesión", algunas de ellas utilizadas como marcadores ocupacionales, aumentará por el tipo de postura, fuerza, repetividad de movimiento o de forma mixta (Benavides et al. 2000; Luttmann et al. 2003; NIOSH 1997; Silverstein 2001)

Por otra parte, la falta de precisión de los requerimientos biomecánicos y la baja experticia de los voluntarios en la ejecución de un gesto técnico pueden estar influenciando el alto grado de variabilidad entre sujetos reflejado en los resultados. Bril y colaboradores (2010) evidencian que en la percusión para la talla de instrumentos, el factor de experticia influye sobre la fuerza, ángulos de ejecutar la técnica, y por ende la precisión del golpe ejecutado para obtener el producto deseado.

De igual manera, el método de electromiografía superficial no permite evaluar directamente el músculo, por lo que no se pueden medir aquellos que están en un nivel más profundo, por otra parte, el tejido adiposo sobre el músculo puede interferir en la lectura de la sEMG. Lo anterior, es lo que no permite diseñar un estudio que permita comparar una muestra de sexo masculino con una femenina, impidiendo entender cómo la variación del desarrollo muscular de hombres y mujeres que pueden estar afectando la reclusión de unidades motoras en cada uno de los gestos técnicos, es sabido que factores hormonales interfieren en la evaluación de los MOA entre hombres y mujeres (Ahtiainen et al. 
2003; Weiss 2003; Weiss et al. 2012, Wilczak 1998) y estudios a nivel muscular han determinado la influencia de la testosterona en el desarrollo de masa muscular, principalmente en su diámetro transverso (Hunter 2000; Clarkson 2003). No obstante lo anterior, las limitantes aquí presentadas no son ajenas a otros estudios similares en el campo de arqueología experimental y del estudio del trabajo realizados en otras partes del mundo y más que presentársenos como barreras analíticas, las vemos como oportunidades para futuras investigaciones, que nos estimulan a seguir desarrollando nuestra línea de investigación.

\section{Conclusión}

Se considera que este estudio es un aporte a la reflexión de la interpretación de indicadores óseos de actividad física, en este caso para contextos arqueológicos. Se ejemplificó cómo la activación muscular se puede ver afectada por la postura adoptada y cómo varia para un mismo gesto técnico el porcentaje de activación muscular.

Creemos que para mejorar la interpretación de indicadores óseos de actividades en las poblaciones pasadas, las investigaciones deben ser acompañadas de estudios cinemáticos complementarios, no sólo desde la revisión bibliográfica de antecedentes médicos de actividades actuales o deportivas, sino con investigaciones que permitan incluir otros componentes socioculturales en la producción del movimiento y precisarlo a su contexto en particular. Dichas investigaciones deberían considerar incluir antecedentes locales, etnográficos y arqueológicos que permitan hacer más preciso el estudio de las actividades desde su dimensión sociocultural, a partir de un enfoque interdisciplinario y no basado netamente en los marcadores óseos de actividades, los cuales deben ser vistos como un complemento más y no un fin en sí mismos.

Finalmente, a pesar de las limitantes inherentes a este tipo de trabajos, se hace inevitable diseñar metodologías que permitan la caracterización -actuales o experimentalde las tareas que son parte de la cadena operativa de la actividad a estudiar no sólo desde la teoría arqueológica y antropológica, creemos que inclusión de metodologías del estudio del trabajo, desarrolladas por la ergonomía o salud laboral, enriquezcan los argumentos y ayudar a la reconstrucción de las actividades pasadas, incluyéndose tanto la dimensión social y también biológica.

Concepción, Chile, 19 de abril 2017

\section{Agradecimientos}

Esta investigación fue financiada por los proyectos FONDART-2014 N³9560, FONDECYT 1110196, FONDECYT 151203.

\section{Referencias}

Appendino, M., M. Giordano y P. Sisto. 2002. "Postura bípeda y cultura", Facultad de Medicina y Ciencias de la Salud, Universidad Abierta Interamericana, Argentina, 108. Seminario para optar al grado de Licenciado en Kinesiología y Fisiatría. http://imgbiblio.vaneduc.edu.ar/fulltext/files/TC043341.pdf

Ahtiainen, J.P., Pakarinen, A., Alen, M., Kraemer, W.J., Häkkinen, K., 2003. Muscle hypertrophy, hormonal adaptations and strength development during strength training in strengthtrained and untrained men. European Journal Applied Physiology, 89: 555-563.

Benavides, F. Ruiz-Frutos y C. García. 2000. Salud Laboral, 385, Masson, Barcelona.

Blanco, J.F., I. Correa, C. Flores y G. Pimentel. 2017. La extracción prehispánica de recursos minerales en el internado QuillaguaCosta, Desierto de Atacama. Estudios atacameños, Epub February 09, 2017.

https://dx.doi.org/10.4067/S0718-10432017005000003

Bracco, J. P., O. Dutour, R. Chenorkian y A. Defleur. 1991. Gestes Techniques et Debitage Experimental. Elements de Reflexion et Potentialites de Recherches dans l'etude du geste en prehistoire. Tecnología y Cadenas Operativas Líticas. U.A.B, Treballs $d^{\prime}$ Arqueologia, $15-18$ : 163-172.

Bril, B., R. Rein, T. Nonaka, F. Wenban-Smith y G. Dietrich. 2010. The role of expertise in tool use: skill differences in functional action adaptations to task constraints. Journal of Experimental Psychology: Human Perception and Performance, 36 (4): 825839.

Capasso, L., K.A.R. Kennedy y C.A. Wilczak. 1999. Atlas of occupational markers on human remains, 183, Journal of Paleopathology-Monographic Publication 3, Italy.

Clarkson, H. 2003. Proceso evaluativo musculoesquelético: amplitud del movimiento articular y test manual de fuerza muscular, 464, Editorial Paidotribo, Barcelona.

D'Errico, F. 1992. Technology, Motion, and the Meaning of Epipaleolithic Art. Current Anthropology, 33 (1): 94-109.

Dutour, O. 1992. Activités physiques et squelette humain: le difficile passage de l'actuel au fossile. Bulletins et Mémoires de la Société d'anthropologie de Paris, Nouvelle Série, 4 (3-4): 233-241.

Fernández, J. M., R. Acevedo y C.B. Tabernig. 2007. Influencia de la fatiga muscular en la señal electromiográfica de músculos estimulados eléctricamente. Revista EIA, 7: 111-119.

Hawkey, D. y H. Merbs. 1995. Activities induced musculoskeletal stress markers (MSM) and subsistence strategy changes among ancient Hudson Bay Eskimos, International Journal Osteoarchaeology, 5: 324-338.

Hunter, G. 2000. Fisiología del Músculo. National Strength and Conditioning Association, Principios del entrenamiento de la fuerza y del acondicionamiento físico, Capítulo 1: 3-14, Editorial Panamericana, Madrid. 
Ingold, T. 2000. The perception of the environment: Essays on livelihood, dwelling and skill, 455, Routledge Press, LondonNew York.

Ingold, T. 2008. Tres en uno: Cómo disolver las distinciones entre mente, cuerpo y cultura- T. Sánchez-Criado. Tecnogénesis: La construcción técnica de las ecologías humanas, Volumen 2, Capítulo 1: 3-33. AlBR. Madrid.

Jurmain, R., F. A. Cardoso, C. Henderson y S. Villotte. 2012. Bioarchaeology's Holy Grail: the reconstruction of activity. A.L. Grauer, A companion to paleopathology, Chapter 29: 531-552, Wiley/Blackwell, Malden.

Kennedy, K. 1989. Skeletal Markers of Occupational Stress. Iscan, M.Y. y K. Kennedy, Reconstruction of Life from the Skeleton, Chapter 8: 129-160, Alan R. Liss, New York.

Luttmann, A., M. Jäger, B. Griefahn, G. Caffier, F. Liebers y U. Steinberg. 2003. Preventing Musculoskeletal Disorders in the Workplace. Protecting Workers' Health Series 5, 40, World Health Organization Publications, India.

http://www.who.int/occupational_health/publications/en/ oehmsd3.pdf (Última Consulta 12/04/2017)

Maulén, J. 2005. "Estudio de fatiga muscular mediante la estimulación de baja frecuencia", Facultad de Medicina, Universidad de Barcelona, 126. Tesis para optar al grado de Doctor en Ciencias Biológicas. http://www.tdx.cat/bitstream/ handle/10803/1129/JMA_TESIS.pdf

Mauss, M. 1934. Les Techniques du corps. Journal de Psychologie, XXXII, 3-4, 15 mars. - 15 avril 1936. Communication présentée à la Société de Psychologie le 17 mai 1934. Colección "Les Clasique des sciences sociales". http://classiques.uqac.ca/ classiques/mauss_marcel/socio_et_anthropo/6_Techniques_ corps/techniques_corps.pdf (Última Consulta 12/04/2017)

Millán, A. 2004. La minería metálica en Chile en el siglo XIX, 88, Editorial Universitaria, Santiago.

Niinimäki, S., L. Härkönen, R. Nikander, S. Abe, C.r Knüsel, H. Sievänen. 2016. The cross-sectional area of the gluteus maximus muscle varies according to habitual exercise loading: Implications for activity-related and evolutionary studies. HOMO - Journal of Comparative Human Biology, 67(2): 125-37.

NIOSH (National Institute for Occupational Safety and Health). 1997. Musculoskeletal Disorders and Workplace Factors - A Critical Review of Epidemiologic Evidence for Work-Related Musculoskeletal Disorders of the Neck, Upper Extremity, and Low Back, 590, NIOSH Publications, Cincinnati.

Parra, S. 2014. "Aproximación funcional y traceología de los instrumentos de molienda del Complejo Huentelauquén". Facultad de Ciencias Sociales, Universidad de Chile, Chile, 149. Memoria para optar al grado de título de Arqueóloga. http:// repositorio.uchile.cl/handle/2250/131781

Pasciuto, I., S. Ausejo, J. T. Celigüeta, A. Suescun y A. Cazón. 2014. A comparison between optimization-based human motion prediction methods:data-based, knowledge-based and hybrid approach. Structural and Multidisciplinary Optimization, 49 (1): 169-183.

Peterson Kendall, F., E. Kendall Mccreary, P. Geise Provance, M. McIntyre Rodgers, y W. A. Romani. 2007. Kendall's Músculos: Pruebas funcionales, postura y dolor, 528, Marban, Madrid.

Salazar, D., D. Jackson, J.L. Guendon, H. Salinas, D. Morata, V. Figueroa, G. Manríquez y V. Castro. 2011. Early Evidence (ca. 12,000 BP) for Iron Oxide Mining on the Pacific Coast of South America. Current Anthropology, 52 (3): 463-475.

Salazar, D., H. Salinas, J. L. Guendon, D. Jackson y V. Figueroa. 2013. Hunter-Gatherers-Fisher Ming During the Archaic Period in Coastal Northern Chile. Tripcevich, N. y K.J. Vaugh, Mining and Quarrying in the Ancient Andes. Sociopolitical, Economic and Symbolic Dimensions, Chapter 7: 37-156, Springer, London.

Salazar, D., y F. Vilches. 2014. La arqueología de la minería en el centro-sur andino: Balance y Perspectivas. Estudios atacameños, 48: 5-21.

Salinas, H. y D. Salazar. 2008. Cadenas operativas y sistemas de explotación minera prehispánica. En D. Jackson, D. Salazar y A. Troncoso, Puentes hacia el pasado: reflexiones teóricas en arqueología, Capítulo 4: 73-92. Serie Monográfica de la Sociedad Chilena de Arqueología 1, Santiago.

Salinas, H., D. Salazar, J. L. Guendon, V. Figueroa y G. Manríquez. 2012. Tecnología Lítica Minera del Período Arcaico en Quebrada San Ramón, Taltal (II Región de Antofagasta. Actas del XVIII Congreso Nacional de Arqueología Chilena, 197-206. Sociedad Chilena de Arqueología, Valparaíso.

Santana Cabrera, J. 2009-2010. Marcadores óseos de actividad física en la población aborigen de Gáldar (siglos XI-XVd.n.e.). Vegueta, 11: 101-122.

Silverstein, B. 2001. Work-related Musculoskeletal Disorders: General Issues. W. Karwowski. International Encyclopedia of Ergonomics and Human Factors, Volume 3, Part 10-Health and Safety: 1621-1624. Taylor \& Francis, London/New York.

Villotte, S. y C. J. Knüsel. 2012. Understanding Entheseal Changes: Definition and Life Course Changes. International Journal of Osteoarchaeoly, 23: 135-146.

Weiss, E. 2003. Understanding Muscle Markers: Aggregation and Construct Validity. American Journal of Physical Anthropology, 121: 230-240.

Weiss, E., L. Corona y B. Schultz. 2012. Sex Differences in Musculoskeletal Stress Markers: Problems with Activity Pattern Reconstructions. International Journal of Osteoarchaeoly, 22: 70-80.

Wilczak, C. A. 1998. Consideration of sexual dimorphism, age, and asymmetry in quantitativemeasurements of muscle insertion sites. International Journal of Osteoarchaeology, 8: 311-325. 